\title{
Fenofibrate improves endothelial function and plasma myeloperoxidase in patients with type 2 diabetes mellitus: an open-label interventional study
}

Cristina Nita ${ }^{1 * \dagger}$, Cornelia Bala ${ }^{1 \dagger}$, Mihai Porojan ${ }^{2}$ and Nicolae Hancu ${ }^{1}$

\begin{abstract}
Background: Fenofibrate offers a number of benefits on the cardiovascular system and it is plausible that its antiinflammatory, anti-oxidant and anti-fibrotic effects and enhancement of cardiac metabolic performances may account for its direct cardioprotective effects.

In this study we aimed to investigate the effect of fenofibrate on endothelial function assesed by vascular studies and levels of soluble E-selectin (sE-selectin) as well as the effect on plasma myeloperoxidase (MPO) in patients with type 2 diabetes mellitus (T2DM) without previous use of lipid-lowering medication.
\end{abstract}

Methods: 27 patients (14 men and 13 women) with T2DM and good glycemic control (HbA1c: min 5.9\%, max: 7.1\%) treated with metformin monotherapy, without previous use of lipid-lowering medication were enrolled in this study. Vascular studies included measures of brachial artery diameter before and after release of a suprasystolic ischemia. FMD was calculated as the percent (\%) change in arterial diameter following reactive hyperemia. Student's paired $t$ test and Wilcoxon Signed Ranks Test were used to compare values before and after fenofibrate therapy.

Results: Fenofibrate therapy significantly increased post ischemia mean brachial artery diameter at $60 \mathrm{~s}$ (from 4.7 $[4.4 ; 5.0] \mathrm{mm}$ to $4.9[4.6 ; 5.2] \mathrm{mm}, \mathrm{p}=0.01$ ) and at $90 \mathrm{~s}$ (from $4.7[4.4 ; 5.0] \mathrm{mm}$ to 4.9 [4.6; 5.1], $\mathrm{p}=0.02$ ). FMD response to hyperaemia at $60 \mathrm{~s}$ increased with $4.5 \pm 13.7 \%$ (median value pre- treatment: $22.2 \%$, median value post- treatment $25.0 \%, z=-2.9, p=0.004$ ). After 8 weeks of fenofibrate therapy, plasma MPO levels decreased to $49.5[30.3 ; 71.5] \mathrm{ng} / \mathrm{ml}$ (\% change from baseline $=4.6 \%, \mathrm{z}=-2.2, \mathrm{p}=0.03$ ) and mean plasma sE-selectin levels decreased to $67.1[54.4 ; 79.8] \mathrm{ng} / \mathrm{ml}$, (\% change from baseline $=2.6 \%, \mathrm{p}=0.03$ ).

Conclusion: In patients with T2DM without previous treatment for dyslipidemia, short-term treatment with fenofibrate improved vascular endothelial function as demonstrated by increased post ischemia mean brachial artery diameter, increased FMD and decreased plasma sE-selectin and favorably affected plasma MPO levels. Therefore, fenofibrate may be considered a protective cardiovascular drug in this group of patients.

Trial registration: (Australian New Zealand Clinical Trials Registry ANZCTR12612000734864)

Keywords: Fenofibrate, Myeloperoxidase, Endothelial dysfunction, sE-selectin, Type 2 diabetes

\footnotetext{
*Correspondence: cnita@umfcluj.ro

${ }^{\dagger}$ Equal contributors

'Department of Diabetes, Nutrition and Metabolic Diseases, "Iuliu Hatieganu" University of Medicine and Pharmacy Cluj-Napoca, 2-4 Clinicilor Street, 400006 Cluj-Napoca, Romania

Full list of author information is available at the end of the article
} 


\section{Background}

Type 2 diabetes mellitus (T2DM) is associated with a high risk of cardiovascular (CV) events, irrespective of the presence of other traditional risk factors [1]. Current evidence suggests the central role of endothelium in all phases of the atherosclerotic process [2]. Most CV risk factors activate molecular pathways that result in increased expression of cytokines and cellular adhesion molecules involved in the adhesion and migration of monocytes into the subendothelial space $[2,3]$, leading to the initiation, progression, and destabilization of the atherosclerotic lesion. E-selectin is a molecule of endothelial origin, which has been associated with carotid atherosclerosis and incident coronary heart disease $[3,4]$. Flow mediated dilation (FMD) evaluates endothelial dependent dilation in the brachial artery after occlusion, a measure of endothelial function in humans $[5,6]$.

Myeloperoxidase (MPO) is a predominantly leukocytederived enzyme, involved in the initiation, destabilization of atherosclerotic plaque and genesis of acute coronary syndromes [7]. Plasma MPO levels have been positively associated with coronary artery disease (CAD) and risk of a subsequent cardiac event [8-11].

Therefore, drugs that have the potential to influence both vascular function and MPO have become highly interesting, especially if already used in the treatment of other CV risk factors in persons with diabetes. Fenofibrate offers a number of benefits on the CV system [12-14] and it is plausible that its anti-inflammatory, anti-oxidant and anti-fibrotic effects and enhancement of cardiac metabolic performances may account for its direct cardioprotective effects [15-17]. Previous studies showed that these effects could be mediated by decreased plasma high sensitivity C-reactive protein, fibrinogen, plasminogen activator inhibitor (PAI)-1 and decreased monocyte cytokine release [15], suppression of thromboxane A2 receptor, cytosolic calcium mobilization, and cyclooxygenase (COX)-1 activity [16] and decrease in inducible nitric oxide synthase, cyclooxygenase (COX)2 and matrix metallopeptidase 9 (MMP-9) [17].

The main purpose of this study was to investigate the role of fenofibrate on endothelial function, assessed by vascular studies and soluble E-selectin (sE-selectin), and on plasma MPO in patients with T2DM without previous use of lipid-lowering medication.

\section{Methods}

\section{Study design and protocol}

This was an interventional, open label study enrolling patients with T2DM treated with metformin alone, and without previous use of lipid-lowering medication. Participants were recruited from a T2DM population in an outpatient clinic from Romania. According to treatment protocols in place in Romania, each patient with diabetes treated with non-insulinic drugs must attend a specialist visit twice a year.

Participants were enrolled between August 1st and September 15, 2012 and followed for 8 weeks.

Each participant attended 3 study visits: screening, visits 1 and 2 ( 8 weeks apart). Starting from visit 1 participants fulfilling inclusion criteria and without any exclusion criteria, received $160 \mathrm{mg}$ fenofibrate/day for 8 weeks. The pills were counted at the end of study; compliance was considered satisfactory if $>90 \%$ of them were taken.

Before and at the end of the study, data were collected on medical history (visit 1), anthropometric variables, blood pressure (BP), FMD of the brachial artery, as well as adverse events (visit 2). Blood samples were collected at screening, visit 1 and 2 in fasting conditions as described below. At visit 1 each participant received instructions to maintain his/her usual nutritional habits and not to modify any drug treatment throughout the study.

The research was conducted in accordance with the guidelines in The Declaration of Helsinki and Good Clinical Practice. Study protocol was approved by the Iuliu Hatieganu University of Medicine and Pharmacy Ethics Committee. Written informed consent was obtained from each participant prior to any study procedures.

\section{Study objectives were to assess}

1. The variation of brachial artery diameters and of FMD response to hyperaemia, after 8 weeks of treatment with fenofibrate compared to pre-treatment values

2. The variation of plasma MPO and soluble sE-selectin after 8 weeks of treatment with fenofibrate as compared with pre-treatment values.

\section{Study population}

We enrolled men and women $\geq 18$ years of age, with T2DM defined according to World Health Organization criteria (WHO) [18], who were on stable doses of metformin for at least 3 months, with glycated haemoglobin (HbA1c) $<7.5 \%$ and without previous treatment with any lipid-lowering drugs.

Exclusion criteria were: known type 1 and other specific types of diabetes (e.g. genetic defects of the $\beta$-cell, genetic defects in insulin action, diseases of the exocrine pancreas, endocrinopathies, drug- or chemical-induced diabetes, infections, uncommon forms of immunemediated diabetes, other genetic syndromes associated with diabetes) according to WHO classification of diabetes mellitus [18], treatment with other hypoglycaemic drugs during the 3 months preceding the screening visit, any acute cardiovascular event within last 3 months, genetic conditions affecting lipids metabolism (e.g. familial hypercholesterolemia, lipoprotein lipase deficiency), 
uncontrolled endocrine or metabolic diseases, chronic kidney disease (glomerular filtration rate [eGFR] $<50 \mathrm{ml} /$ $\min / 1.73 \mathrm{~m}^{2}$, contraindicating treatment with fenofibrate), serum triglyceride level $\geq 400 \mathrm{mg} / \mathrm{dl}$, hepatic enzymes $>3$ upper normal limits (UNL), history of alcohol abuse, changes in antihypertensive therapy during the last 3 months.

\section{Data collection and laboratory tests}

A complete medical history, including diabetes duration and treatment, was obtained for each participant; height, weight and waist circumference were determined by a standardized protocol. Waist circumference was measured at half of the distance between the lower border of the last rib and the upper border of the iliac crest at the end of a normal expiration, using a non-stretchable tape measure. Body mass index (BMI) was calculated as weight $(\mathrm{kg}) /[\text { height }(\mathrm{m})]^{2}$. Blood pressure was measured using a calibrated standard mercury sphygmomanometer. All readings were taken after a 5-min rest, in the sitting position.

Fasting blood samples were collected in the morning after an $8 \mathrm{~h}$ overnight fasting period. Fasting plasma glucose, HbA1c, total cholesterol, high-density lipoprotein (HDL) cholesterol, triglycerides, creatinine, hepatic enzymes were determined by automated chemistry analyzer Konelab 30, Thermo Fisher Scientific Inc, Finland. Lowdensity lipoprotein (LDL) cholesterol was calculated using Friedewald formula [19]. eGFR was estimated using online available Modification of Diet in Renal Disease (MDRD) calculator (http://www.nephron.com/MDRD_GFR.cgi). sEselectin and plasma MPO were measured in plasma (EDTA) using commercially available enzyme-linked immunosorbent assay kits for quantitative detection (DRG Diagnostics GmbH, Germany and DRG International, Inc, USA) on an automated ELISA reader (Tecan).

Adverse events were recorded throughout of study. Safety parameters included serum creatinine, eGFR, and hepatic enzymes.

\section{Vascular studies}

Imaging studies of the right brachial artery were performed in fasting condition before and after 8 weeks of fenofibrate therapy with a high-resolution, ultrasound imaging system (Aloka ProSound Alpha 10 Premier, Aloca Co., Ltd.) using M-mode, and electrocardiogramtriggered ultrasound images obtained with a $10 \mathrm{MHz}$ linear-array transducer. All vascular studies were performed according to a protocol previously described by Celermajer and co-workers [5], by measuring the arterial response to reactive hyperemia. Studies were performed at $20-24^{\circ} \mathrm{C}$ in a dark, quiet room. The study participant rested for at least $10 \mathrm{~min}$ before the first scan and remained in a recumbent position throughout the investigation. Scanning of the brachial artery was performed 3-10 cm above the elbow, at baseline (before ischemia) and at 45, 60, 90, and $120 \mathrm{~s}$ after release of a suprasystolic ischemia of the forearm with a pneumatic cuff, inflated at $250 \mathrm{mmHg}$ for $4 \mathrm{~min}$. Images were taken at the end of the diastole and digitalized. Four cardiac cycles were analyzed at the end of the diastole, and arterial diameter was automatically measured using special software (E-tracking) and then averaged. FMD was calculated as the percent (\%) change in arterial diameter following reactive hyperemia when compared to the baseline diameter.

\section{Statistical analysis}

Statistical analysis was performed using SPSS-PC 15.0 (SPSS Inc., Chicago, IL, USA). Data were considered to be normally distributed if the ratio of skewness to its standard deviation did not exceed the value of 2. Data were expressed as a mean [95\% Confidence Interval] for variables with normal distribution, median [1st quartile; 3rd quartile] for variables with abnormal distribution or frequency for dichotomial variables. Student's paired t test (for variables with normal distribution) and Wilcoxon Signed Ranks Test (for variables with deviations from normal distribution) were used to compare values before and after fenofibrate therapy. Correlations were assessed with Pearson's and Spearman's correlation coefficient analysis. The level of significance was set at 0.05 , and all tests were performed two- sided.

\section{Results}

\section{Baseline characteristics}

Twenty seven patients (14 men and 13 women) fulfilling the inclusion criteria and without any exclusion criteria were enrolled in this study. Two participants (one male and one female) did not complete the study: one was excluded from the study due to poor compliance with fenofibrate treatment and one dropped out soon after fenofibrate treatment initiation for personal reasons and refused to present for reevaluation. Mean duration of diabetes was 3.8 years. All participants had good glycemic control (HbA1c range: 5.9\%-7.1\%). Doses of metformin ranged from 850 to $3000 \mathrm{mg} /$ day. $76.0 \%$ of study participants had a history of hypertension and $20.0 \%$ had cardiovascular diseases. Characteristics of study participants are presented in Table 1.

Compared with baseline, at visit 3 participants presented significantly lower levels of total cholesterol $(-24.3 \mathrm{mg} / \mathrm{dl}, \mathrm{p}=0.002)$, triglycerides levels $(-54.9 \mathrm{mg} /$ $\mathrm{dl}, \mathrm{p}<0.001)$, systolic and diastolic blood pressure $(-6.8 \mathrm{mmHg}, \mathrm{p}=0.04$ and $-3.8 \mathrm{mmHg}, \mathrm{p}=0.02$, respectively). No statistically significant effect was observed on HDL-cholesterol and on LDL-cholesterol $(\mathrm{p}>0.05)$. In addition, no change was observed in fasting plasma 
Table 1 Characteristics of the 25 patients at baseline and at follow up (8 weeks of treatment)

\begin{tabular}{|c|c|c|c|}
\hline Variables & Visit 1 (Pre-treatment) & Visit 2 (Post treatment) & $\mathbf{p}$ \\
\hline Age (years) & $59.2[55.2 ; 63.2]$ & - & \\
\hline Male gender (\%) & $13.0(52.0 \%)$ & - & \\
\hline Current smoker & $4.0(16.0 \%)$ & - & \\
\hline Hypertension & $19.0(76.0 \%)$ & - & \\
\hline Cardiovascular disease & $5.0(20.0 \%)$ & - & \\
\hline Ischemic heart disease & $4.0(16.0 \%)$ & - & \\
\hline Ischemic stroke & $1.0(4 \%)$ & - & \\
\hline \multicolumn{4}{|l|}{ Medication } \\
\hline ACEI & $10.0(40.0 \%)$ & - & \\
\hline$\beta$-Adrenergic receptor blocker & $8.0(32.0 \%)$ & - & \\
\hline Diuretics & $8.0(32.0 \%)$ & - & \\
\hline Calcium channel blocker & $7.0(28.0 \%)$ & - & \\
\hline Angiotensin receptor blocker & $4.0(16.0 \%)$ & - & \\
\hline Aspirin & $7.0(28.0 \%)$ & - & \\
\hline $\mathrm{SBP}(\mathrm{mmHg})$ & 149.8 [143.5; 156.1] & $142.7[137.6 ; 147.8]$ & 0.04 \\
\hline $\mathrm{DBP}(\mathrm{mmHg})$ & $91.5[87.4 ; 95.5]$ & $87.5[82.8 ; 92.2]$ & 0.02 \\
\hline BMI $\left(\mathrm{kg} / \mathrm{m}^{2}\right)$ & $28.7[26.6 ; 34.2]^{a}$ & $28.7[26.7 ; 34.0]^{a}$ & 0.79 \\
\hline Waist (cm) & 108.5 [104.0; 113.0] & $110.4[105.5 ; 115.3]$ & 0.06 \\
\hline FPG (mg/dl) & 136.9 [129.0; 144.8] & $137.6[129.7 ; 145.5]$ & 0.82 \\
\hline HbA1c (\%) & $6.5[6.3 ; 6.8]$ & $6.5[6.3 ; 6.7]$ & 0.53 \\
\hline \multicolumn{4}{|l|}{ Lipids } \\
\hline Total cholesterol (mg/dl) & $182.0[166.0 ; 220.0]^{a}$ & $168.0[151.0 ; 192.5]^{a}$ & 0.01 \\
\hline HDL cholesterol (mg/dl) & $45.0[40.1 ; 50.0]$ & $44.1[37.7 ; 50.5]$ & 0.88 \\
\hline LDL-cholesterol (mg/dl) & $104.0[93.0 ; 139.3]^{a}$ & $102.0[89.1 ; 125.6]^{a}$ & 0.14 \\
\hline Triglycerides (mg/dl) & $168.6[140.2 ; 197.0]$ & $114.1[95.4 ; 132.8]$ & $<0.001$ \\
\hline eGFR (ml/min/1.73 m²) & $107.7[93.4 ; 122.0]$ & $96.8[82.6 ; 111.1]$ & 0.007 \\
\hline TGO (IU/L) & $20.0[15.5 ; 25.0]^{a}$ & $22.0[18.0 ; 26.0]^{a}$ & 0.10 \\
\hline TGP (IU/L) & $22.0[17.0 ; 27.0]^{a}$ & $24.0[20.0 ; 28.0]^{a}$ & 0.48 \\
\hline
\end{tabular}

Data in table are presented as mean [95\% $\mathrm{Cl}$ ] for continuous variables, median [1st quartile; 3rd quartile] for variables with abnormal distribution and number (\%)

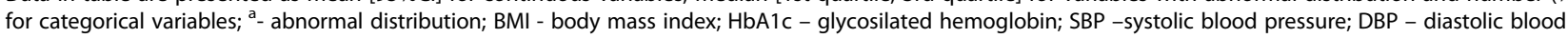
pressure; FPG - fasting plasma glucose; EGFR- estimated glomerular filtration rate.

glucose, HbA1c, BMI and waist after fenofibrate treatment (Table 1, p >0.05).

\section{FMD}

The variation of brachial artery diameters before and after treatment is shown in Figure 1. Mean basal brachial artery diameter was similar at each ultrasound assessment, with no significant difference between pre-treatment and post-treatment diameter $(\mathrm{p}=0.34)$. Post ischemia mean brachial artery diameter at $60 \mathrm{~s}$ and $90 \mathrm{~s}$ increased significantly after fenofibrate therapy: from $4.7[4.4 ; 5.0] \mathrm{mm}$ to $4.9[4.6 ; 5.2] \mathrm{mm}$ at $60 \mathrm{~s}, \mathrm{p}=0.01$ and from $4.7[4.4 ; 5.0]$ $\mathrm{mm}$ to 4.9 [4.6; 5.1] at $90 \mathrm{~s}, \mathrm{p}=0.02$ ). No significant differences between pre-treatment and post-treatment diameter were observed at $45 \mathrm{~s}$ and $120 \mathrm{~s}(\mathrm{p}=0.18$ and 0.67 , respectively).
Additionally, compared with pre-treatment values, FMD response to hyperaemia at $60 \mathrm{~s}$ increased with $4.5 \pm 13.7 \%$ (median value pre- treatment: $22.2 \%$, median value posttreatment $25.0 \%, \mathrm{z}=-2.9, \mathrm{p}=0.004$ ) (Figure 2).

No correlations were observed between changes in FMD and changes in total cholesterol (Spearman correlation coefficient $\rho=-0.1, \mathrm{p}=0.50)$, triglycerides $(\rho=-0.3$, $\mathrm{p}=0.07)$ or changes in systolic BP $(\rho=-0.2, \mathrm{p}=0.37)$ or diastolic BP $(\rho=-0.08, \mathrm{p}=0.72)$.

\section{MPO and $s E-$-selectin}

At baseline, MPO values ranged from 16.5 to $236.5 \mathrm{ng} / \mathrm{ml}$, with a median level of $55.0[38.5 ; 85.3] \mathrm{ng} / \mathrm{ml}$ and values of sE- selectin ranged from 15.0 to $142.0 \mathrm{ng} / \mathrm{ml}$, with a mean level of 75.2 [59.9; 90.5] ng/ml. After 8 weeks of fenofibrate therapy, plasma MPO levels decreased to 49.5 


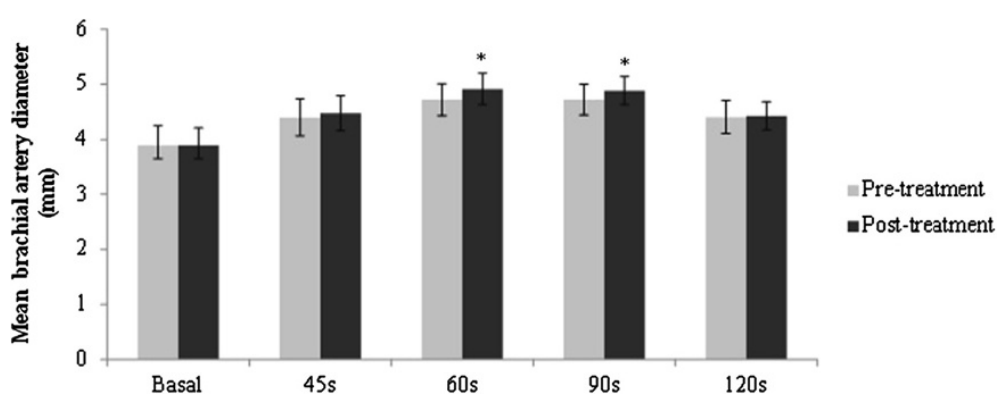

Figure 1 Effect of fenofibrate on brachial artery diameter in basal conditions and during hyperemia at 45, 60, 90 and 120 seconds. *Statistically significant difference in mean brachial artery diameter pre- and post-treatment. Error bars represent $95 \%$ confidence intervals Basal, before hyperemia; $45 \mathrm{~s}$, hyperemia $45 \mathrm{~s} ; 60 \mathrm{~s}$, hyperemia $60 \mathrm{~s} ; 90 \mathrm{~s}$, hyperemia $90 \mathrm{~s}$; $120 \mathrm{~s}$, hyperemia $120 \mathrm{~s}$.

$[30.3 ; 71.5] \mathrm{ng} / \mathrm{ml}$ (\% change from baseline $=4.6 \%, \mathrm{z}=$ $-2.2, \mathrm{p}=0.03$ ) (Figure 3) and mean plasma sE-selectin levels decreased to $67.1[54.4 ; 79.8] \mathrm{ng} / \mathrm{ml}$ (\% change from baseline $=2.6 \%, \mathrm{p}=0.03$ ) (Figure 4$)$.

\section{MPO and endothelial dysfunction}

No correlation was observed between \% change in MPO levels and \% change in FMD (Spearman correlation coefficient $\rho=0.3, p=0.18$ ) or between \% change in MPO levels and \% change in E selectin (Spearman correlation coefficient $\rho=-0.2, \mathrm{p}=0.29$ ).

\section{Safety parameters}

No serious adverse effects were observed throughout the study, and none of the patients withdrew due to adverse effects. A statistically significant decrease of eGFR occurred between baseline and final visit $(107.7$ [93.4; 122.0] vs. $96.8[82.6 ; 111.1] \mathrm{ml} / \mathrm{min} / 1.73 \mathrm{~m}^{2}, \mathrm{p}=0.007$ ) (Table 1 ).

\section{Discussions}

In this study, we showed that fenofibrate improved endothelial dysfunction in T2DM patients without previous use of lipid-lowering medication and that this

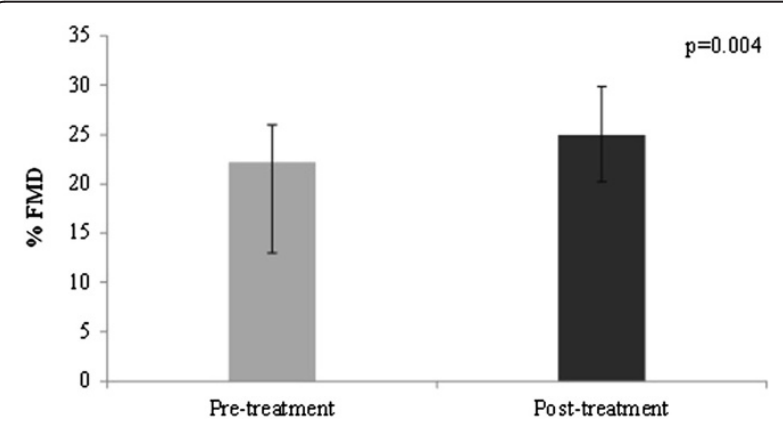

Figure 2 Effect of fenofibrate on percent flow-mediated dilation. Bars represent median values; error bars represent quartile 1 and quartile 3\% FMD, percent flow-mediated dilation. improvement was not related to changes of the lipid profile or blood pressure. We also showed that fenofibrate reduced plasma MPO concentrations.

Our results demonstrated that short term treatment with fenofibrate significantly decreased total cholesterol and triglycerides concentrations. Additionally, we showed that it significantly improved post ischemia brachial artery diameter and FMD, an effect which was independent of the reduction in plasma lipids and blood pressure, decreased plasma MPO and sE-selectin levels and BP values.

\section{Effect of fenofibrate on lipid profile}

Regarding the effect of fenofibrate on plasma triglycerides and total cholesterol, our results are consistent with those of large clinical trials, showing that fenofibrate therapy in patients with dyslipidemia is associated with significant reductions of triglycerides, total and LDLcholesterol levels, and increases HDL-cholesterol levels [12,20-25]. We did not observe any improvement in LDL and HDL- cholesterol levels. This may be explained by the normal lipid levels observed in most of the participants enrolled in our study.

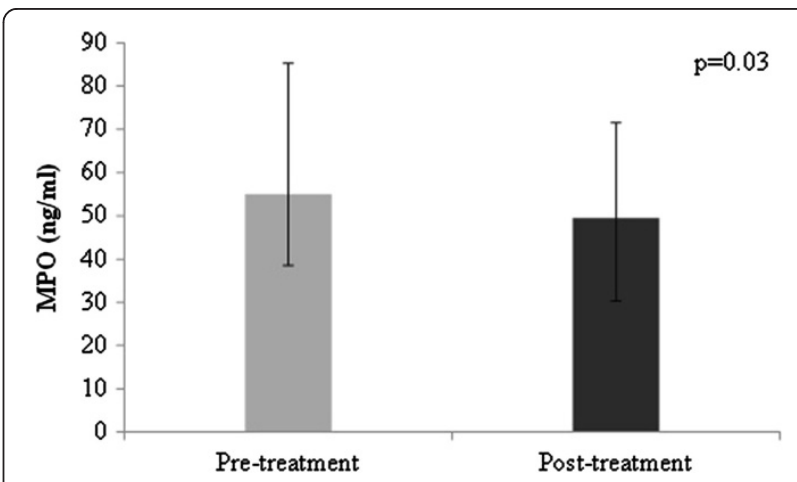

Figure 3 Myeloperoxidase levels $(\mathrm{ng} / \mathrm{ml}$ ) before and after treatment with fenofibrate. Bars represent median values; error bars represent quartile 1 and quartile $3 \mathrm{MPO}$, myeloperoxidase. 


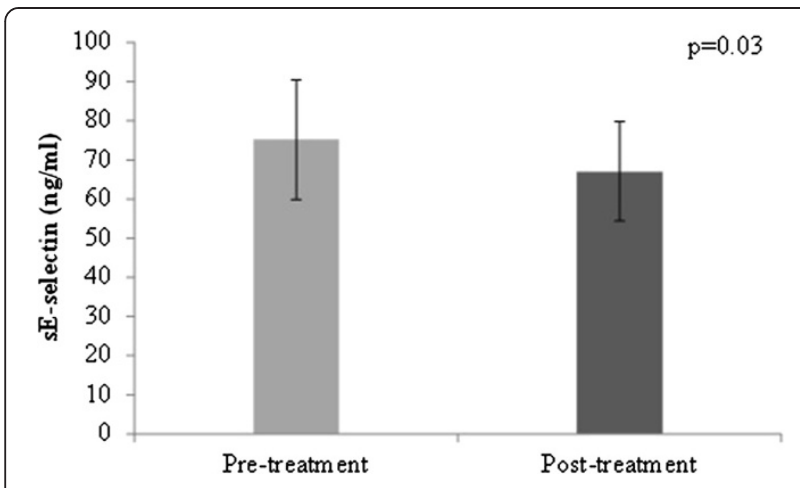

Figure $4 \mathrm{sE}$-selectin levels $(\mathrm{ng} / \mathrm{ml}$ ) before and after treatment with fenofibrate. Error bars represent $95 \%$ confidence intervals.

\section{Effect of fenofibrate on blood pressure}

The results of our study showed a small but significant reduction in both systolic and diastolic BP. In several animal models it was shown that fenofibrate may have a favorable effect on this parameter. Shatara et al. demonstrated that fenofibrate reduced blood pressure in stroke-prone spontaneously hypertensive rats and Dahl salt-sensitive rats [26]. Also, fenofibrate prevented the development of hypertension, myocardial inflammation and fibrosis in Ang-II-infused rats [27]. However, no data in humans have been published.

\section{Effect of fenofibrate on vasomotor function (FMD and sE-selectin)}

The results of our study revealed that fenofibrate therapy significantly improved post ischemia brachial artery diameter and \%FMD. This finding is consistent with other studies that have shown significantly improved FMD response to hyperemia in patients with metabolic syndrome or hypertriglyceridemia following 6-8 weeks of fenofibrate administration [28-30]. A similar effect, partially related to enhanced reduction in LDL-cholesterol and apoB-100 concentrations, has been shown in T2DM patients treated with statins [31]. It was also observed that short-term treatment with fenofibrate improves vascular endothelial function in healthy normolipidemic middleaged and older adults by reducing oxidative stress and induces an increase in endothelial nitric oxide synthase [13].

A favorable impact of 8-week treatment with fenofibrate on FMD in T2DM patients with typical or mixed dyslipidemia has recently been reported [32], and this effect was independent of reduction in lipid parameters. These findings are concordant with our results, suggesting that in patients with T2DM fenofibrate could have positive effects on endothelial dysfunction beyond its antihyperlipidemic action. It cannot be excluded that more subtle changes such as LDL cholesterol particle size could be, at least in part, responsible for improvement in endothelial function with fenofibrate.

We cannot completely exclude that changes in systolic and diastolic BP had an impact on FMD, despite lacking correlations between the three parameters. The interpretation of this possible relationship is limited by the study design which was not placebo-controlled.

Regarding the improvement in sE-selectin levels seen in our study, the results are discordant with those derived from the Fenofibrate Intervention and Event Lowering in Diabetes (FIELD) trial and other studies showing that both long-term and short-term fenofibrate treatment have no effect on this marker of arterial damage [33,34].

Observational studies have suggested that there is a strategic accumulation of MPO at the interface between endothelium and media. This observation led to the hypothesis that MPO could affect vascular tone by depleting endothelium derived NO [35,36]. Subendothelial accumulated MPO can act locally as an NO oxidase, inhibiting NO function and causing endothelial dysfunction [37]. The recently published work of Rudolph et al. demonstrated that MPO elicits profound effects on vascular tone of conductance and resistance vessels both in humans, and in animal models [38]. Their results showed a significant inverse correlation between FMD and MPO plasma levels prior to and following nicotine application $(\mathrm{r}=-20.33, \mathrm{P}=0.03 ; \mathrm{r}=-20.30, \mathrm{P}=0.04)$ [38]. Another research conducted by Vita et al. suggested that there was a strong, independent relation between serum MPO level and endothelial dysfunction, as reflected by brachial artery flow-mediated dilation [39]. By contrast, we observed that there was no correlation between \% changes in MPO levels and \% change in FMD or \% changes in E selectin, suggesting that changes in FMD and $\mathrm{E}$ selectin were independent from changes in MPO levels and could be directly attributed to fenofibrate therapy.

A possible explanation for the favorable effects of fenofibrate on endothelial function in T2DM, independent of its antihiperlipidemic action, is that this drug has direct anti-inflammatory and anti-oxidant effects on vascular wall, a hypothesis that was partially confirmed in healthy normolipidemic subjects [13] but not yet tested in patients with diabetes.

\section{Effect of fenofibrate on plasma myeloperoxidase}

To our knowledge, no prior human study has examined the effect of fenofibrate on plasma MPO concentration. Given that MPO adversely influences LDL atherogenic properties and HDL functionality, it may be hypothesized that drugs reducing this enzyme level could have certain benefits in reducing the risk for atherosclerotic events.

Experimental and clinical studies provide support for a potential impact of statin therapy on tissue and plasma 
MPO levels both in animal models and in humans [40-43]. In clinical studies, statins reduced circulating levels of MPO in patients with congestive heart failure [40], acute coronary syndromes [41] and patients with diabetes on dialysis [42]. The study conducted by Ndrepepa et al. demonstrated an association between therapy with beta-blockers on admission and reduced circulating levels of plasma MPO which was independent of cardiovascular risk factors, clinical characteristics and concomitant therapy in a consecutive series of patients with symptomatic CAD [43].

If our results regarding the beneficial effect of fenofibrate on plasma MPO and on endothelial function will be confirmed in larger populations of patients with T2DM, this drug could become a valuable option for reducing atherosclerotic process in this group of high cardiovascular risk patients as part of an early and comprehensive preventive strategy.

\section{Safety issues}

Our results regarding the decline in renal function are concordant with those in the FIELD and Action to Control Cardiovascular Risk in Diabetes (ACCORD) studies showing that fenofibrate caused an acute, sustained plasma creatinine increase [25,44]. In a recent FIELD sub study, Davies et al. assessed fenofibrate's renal effects and found no evidence that the initial increase in plasma creatinine levels represented true renal injury, a finding that has important implications for clinical care [45]. Moreover, it seems that despite initial and reversible increase in plasma creatinine levels, fenofibrate may delay albuminuria and GFR impairment in T2DM patients [46].

\section{Limitations of the study}

The main limitations of the present study are the relatively small number of patients included and the short duration of the fenofibrate treatment.

\section{Conclusions}

In patients with T2DM without previous treatment for dyslipidemia, short-term treatment with fenofibrate improved vascular endothelial function as demonstrated by increased post ischemia mean brachial artery diameter, increased FMD and decreased plasma sE-selectin and favorably affected plasma MPO levels. Therefore, fenofibrate may be considered a protective cardiovascular drug in this group of patients.

\section{Abbreviations}

BMl: Body mass index; BP: Blood pressure; CAD: Coronary artery disease: CV: Cardiovascular; eGFR: Estimated glomerular filtration rate; FMD: Flow mediated dilation; HbA1c: glycated hemoglobin; HDL: High density lipoprotein; LDL: Low density lipoprotein; MPO: Myeloperoxidase; NO: Nitric oxide; sEselectin: Soluble eselectin; T2DM: Type 2 diabetes mellitus; WHO: World Health Organization.

\section{Competing interests}

The authors declare that they have no competing interests.

\section{Authors' contributions}

CN and CB were study coordinators, participated in the design of the study, were responsible for individual selection and characterization, carried out the statistical analysis and drafted the manuscript. MP carried out vascular studies and participated to manuscript preparation. $\mathrm{NH}$ participated in the design of the study and to manuscript preparation. All authors read and approved the final manuscript.

\section{Acknowledgements}

The authors gratefully acknowledge Adriana Rusu (MD, PhD, Teaching Assistant, "Iuliu Hatieganu" University of Medicine and Pharmacy) for her assistance with statistical analysis. We also would like to thank Virginia Baciu (MD, Synlab Laboratory Cluj Napoca) and Mihai Goia (MD, Emergency County Hospital Cluj) for technical assistance.

\section{Author details}

'Department of Diabetes, Nutrition and Metabolic Diseases, "luliu Hatieganu" University of Medicine and Pharmacy Cluj-Napoca, 2-4 Clinicilor Street, 400006 Cluj-Napoca, Romania. ${ }^{2}$ Department of Internal Medicine, "Iuliu Hatieganu" University of Medicine and Pharmacy Cluj-Napoca, 4-6 Clinicilor Street, 400006 Cluj-Napoca, Romania.

Received: 8 October 2013 Accepted: 13 February 2014

Published: 4 March 2014

\section{References}

1. Grundy SM, Howard B, Smith S Jr, Eckel R, Redberg R, Bonow RO: Prevention Conference IV: Diabetes and Cardiovascular disease: Executive Summary Conference Proceeding for Healthcare Professionals from a Special Writing Group of the American Heart Association. Circulation 2002, 105:2231-2239.

2. Tousoulis D, Charakida M, Stefanadis C: Endothelial function and inflammation in coronary artery disease. Heart 2006, 92:441-444.

3. Deanfield JE, Halcox JP, Rabelink TJ: Endothelial function and dysfunction: testing and clinical relevance. Circulation 2007, 115:1285-1295.

4. Hwang SJ, Ballantyne CM, Sharrett AR, Smith LC, Davis CE, Gotto AM Jr, Boerwinkle E: Circulating adhesion molecules VCAM-1, ICAM-1, and E-selectin in carotid atherosclerosis and incident coronary heart disease cases: the Atherosclerosis Risk In Communities (ARIC) study. Circulation 1997, 96:4219-4225.

5. Celermajer DS, Sorensen KE, Gooch VM, Spiegelhalter DJ, Miller OI, Sullivan ID, Lloyd JK, Deanfield JE, Spiegelhalter DJ: Non-Invasive detection of endothelial dysfunction in children and adults at risk of atherosclerosis. Lancet 1992, 340:1111-1115.

6. Dijkhorst-Oei LT, Stroes ESG, Koomans HA, Rabelink TJ: Acute simultaneous stimulation of nitric oxide and oxygen radicals by angiotensin II in humans in vivo. J Cardiovasc Pharmacol 1999, 33:420-424.

7. Nicholls SJ, Zheng L, Hazen SL: Formation of dysfunctional high-density lipoprotein by myeloperoxidase. Trends Cardiovasc Med 2005, 15:212-219.

8. Brennan ML, Penn MS, Van Lente F, Nambi V, Shishehbor MH, Aviles RJ, Goormastic M, Pepoy ML, McErlean ES, Topol EJ, Nissen SE, Hazen SL: Prognostic value of myeloperoxidase in patients with chest pain. $N$ Engl J Med 2003, 349:1595-1604.

9. Zhang R, Brennan ML, Fu X, Aviles RJ, Pearce GL, Penn MS, Topol EJ, Sprecher DL, Hazen SL: Association between myeloperoxidase levels and risk of coronary artery disease. JAMA 2001, 286:2136-2142.

10. Baldus S, Heeschen C, Meinertz T, Zeiher AM, Eiserich JP, Münzel T, Simoons ML, Hamm CW, CAPTURE Investigators: Myeloperoxidase serum levels predict risk in patients with acute coronary syndromes. Circulation 2003, 108:1440-1445.

11. Meuwese MC, Stroes ES, Hazen SL, Van Miert JN, Kuivenhoven JA, Schaub RG, Wareham NJ, Luben R, Kastelein JJ, Khaw KT, Boekholdt SM: Serum myeloperoxidase levels are associated with the future risk of coronary artery disease in apparently healthy individuals: the EPIC-Norfolk Prospective Population Study. J Am Coll Cardiol 2007, 50:159-165.

12. Zambon A, Cusi K: The role of fenofibrate in clinical practice. Diab VasC Dis Res 2007, 4(Suppl 4):S15-S20.

13. Walker AE, Kaplon RE, Lucking SM, Russell-Nowlan MJ, Eckel RH, Seals DR: Fenofibrate improves vascular endothelial function by reducing 
oxidative stress while increasing endothelial nitric oxide synthase in healthy normolipidemic older adults. Hypertension 2012, 60:1517-1523.

14. Keating GM: Fenofibrate: a review of its lipid-modifying effects in dyslipidemia and its vascular effects in type 2 diabetes mellitus. Am $J$ Cardiovasc Drugs 2011, 11:227-247.

15. Pruski M, Krysiak R, Okopien B: Pleiotropic action of short-term metformin and fenofibrate treatment, combined with lifestyle intervention, in type 2 diabetic patients with mixed dyslipidemia. Diabetes Care 2009, 32:1421-1424

16. Lee JJ, Jin YR, Yu JY, Munkhtsetseg T, Park ES, Lim Y, Kim TJ, Pyo MY, Hong JT, Yoo HS, Kim Y, Yun YP: Antithrombotic and antiplatelet activities of fenofibrate, a lipid-lowering drug. Atherosclerosis 2009, 206:375-382.

17. Chen XR, Besson VC, Palmier B, Garcia Y, Plotkine M, Marchand-Leroux C: Neurological recovery-promoting, anti-inflammatory, and anti-oxidative effects afforded by fenofibrate, a PPAR alpha agonist, in traumatic brain injury. J Neurotrauma 2007, 24:1119-1131.

18. World Health Organization: Definition, diagnosis and classification of diabetes mellitus and its complications. Report of a WHO consultation, Part 1: Diagnosis and classification of diabetes mellitus. Geneva: World Health Organization: 1999.

19. Friedewald WT, Levy Rl, Friedrickson D: Estimation of the concentration of Low-density lipoprotein cholesterol in plasma, without use of the preparative ultracentrifuge. Clin Chem 1972, 18:499-502

20. Najib J: Fenofibrate in the treatment of dyslipidemia: a review of the data as they relate to the new suprabioavailable tablet formulation. Clin Ther 2002, 24:2022-2050.

21. Gotto AM Jr, Amarenco P, Assman G, Carmena R, Davignon J, Fruchart J-C, Kastelein JJP, Paoletti R, Tonkin A: The ILIB lipid handbook for clinical practice: dyslipidemia and coronary heart disease. 3rd edition. New York: International Lipid Information Bureau; 2003.

22. McKeage K, Keating GM: Fenofibrate: a review of its use in dyslipidaemia. Drugs 2011, 71:1917-1946.

23. Watts GF, Karpe F: Why, when and how should hypertriglyceridemia be treated in the high-risk cardiovascular patient? Expert Rev Cardiovasc Ther 2011, 9:987-997.

24. Costa J, Borges M, David C, Vaz Carneiro A: Efficacy of lipid lowering drug treatment for diabetic and non-diabetic patients: meta-analysis of randomised controlled trials. BMJ 2006, 332:1115-1124.

25. Keech A, Simes RJ, Barter P, Best J, Scott R, Taskinen MR, Forder P, Pillai A, Davis T, Glasziou P, Drury P, Kesäniemi YA, Sullivan D, Hunt D, Colman P, d'Emden M, Whiting M, Ehnholm C, Laakso M, FIELD study investigators: Effect of long-term fenofibrate therapy on cardiovascular events in 9795 people with type 2 diabetes mellitus (the FIELD study): randomised controlled trial. Lancet 2005, 366:1849-1861.

26. Shatara RK, Quest DW, Wilson TW: Fenofibrate lowers blood pressure in two genetic models of hypertension. Can J Physiol Pharmacol 2000 78:367-371.

27. Diep QN, Benkirane K, Amiri F, Cohn JS, Endemann D, Schiffrin EL: PPAR alpha activator fenofibrate inhibits myocardial inflammation and fibrosis in angiotensin II-infused rats. J Mol Cell Cardiol 2004, 36:295-304.

28. Kon KK, Yeal Ahn J, Hwan Han S, Kyu Jin D, Sik Kim H, Cheon Lee K, Kyun Shin E, Sakuma I: Effects of fenofibrate on lipoproteins, vasomotor function, and serological markers of inflammation, plaque stabilization, and hemostasis. Atherosclerosis 2004, 174:379-383.

29. Koh KK, Han SH, Quon MJ, Ahn JY, Shin EK: Beneficial effects of fenofibrate to improve endothelial dysfunction and raise adiponectin levels in patients with primary hypertriglyceridemia. Diabetes Care 2005, 28:1419-1424.

30. Kilicarslan A, Yavuz B, Guven GS, Atalar E, Sahiner L, Beyazit Y, Kekilli M, Ozer N Oz G, Haznedarog lu IC, Sozen T: Fenofibrate improves endothelial function and decreases thrombin-activatable fibrinolysis inhibitor concentration in metabolic syndrome. Blood Coagul Fibrinolysis 2008, 19:310-314.

31. Hamilton SJ, Chew GT, Davis TM, Watts GF: Fenofibrate improves endothelial function in the brachial artery and forearm resistance arterioles of statin-treated Type 2 diabetic patients. Clin Sci (Lond) 2010, 118:607-615.

32. Ghani RA, Bin Yaakob I, Wahab NA, Zainudin S, Mustafa N, Sukor N, Wan Mohamud WN, Kadir KA, Kamaruddin NA: The influence of fenofibrate on lipid profile, endothelial dysfunction, and inflammatory markers in type 2 diabetes mellitus patients with typical and mixed dyslipidemia. J Clin Lipidol 2013, 7:446-453.
33. Hiukka A, Westerbacka J, Leinonen ES, Watanabe H, Wiklund O, Hulten LM, Salonen JT, Tuomainen TP, Yki-Järvinen H, Keech AC, Taskinen MR: Longterm effects of fenofibrate on carotid intima-media thickness and augmentation index in subjects with type 2 diabetes mellitus. J Am Coll Cardiol 2008, 52:2190-2197

34. Skrha J, Stulc T, Hilgertová J, Weiserová H, Kvasnicka J, Ceska R: Effect of simvastatin and fenofibrate on endothelium in Type 2 diabetes. Eur $J$ Pharmacol 2004, 493:183-189.

35. Abu-Soud HM, Hazen SL: Nitric oxide is a physiological substrate for mammalian peroxidases. J Biol Chem 2000, 275:37524-37532.

36. Eiserich JP, Baldus S, Brennan ML, Ma W, Zhang C, Tousson A, Castro L, Lusis AJ, Nauseef WM, White CR, Freeman BA: Myeloperoxidase, a leukocyte-derived vascular NO oxidase. Science 2002, 296:2391-2394.

37. van der Veen BS, De Winther MP, Heeringa P: Myeloperoxidase: molecular mechanisms of action and their relevance to human health and disease. Antioxid Redox Signal 2009, 11:2899-2937.

38. Rudolph TK, Wipper S, Reiter B, Rudolph V, Coym A, Detter C, Lau D, Klinke A Friedrichs K, Rau T, Pekarova M, Russ D, Knöll K, Kolk M, Schroeder B, Wegscheider K, Andresen H, Schwedhelm E, Boeger R, Ehmke H, Baldus S: Myeloperoxidase deficiency preserves vasomotor function in humans. Eur Heart J 2012, 33:1625-1634.

39. Vita JA, Brennan ML, Gokce N, Mann SA, Goormastic M, Shishehbor MH, Penn MS, Keaney JF Jr, Hazen SL: Serum myeloperoxidase levels independently predict endothelial dysfunction in humans. Circulation 2004, 110:1134-1139.

40. Andreou I, Tousoulis D, Miliou A, Tentolouris C, Zisimos K, Gounari P, Siasos G Papageorgiou N, Papadimitriou CA, Dimopoulos MA, Stefanadis C: Effects of rosuvastatin on myeloperoxidase levels in patients with chronic heart failure: a randomized placebo-controlled study. Atherosclerosis 2010, 210:194-198.

41. Zhou T, Zhou SH, Qi SS, Shen XQ, Zeng GF, Zhou HN: The effect of atorvastatin on serum myeloperoxidase and CRP levels in patients with acute coronary syndrome. Clin Chim Acta 2006, 368:168-172.

42. Stenvinkel $P$, Rodríguez-Ayala E, Massy ZA, Qureshi AR, Barany P, Fellström B, Heimburger $\mathrm{O}$, Lindholm B, Alvestrand A: Statin treatment and diabetes affect myeloperoxidase activity in maintenance hemodialysis patients. Clin J Am Soc Nephrol 2010, 1:281-287.

43. Ndrepepa G, Braun S, Schömig A, Kastrati A: Impact of therapy with statins, beta-blockers and angiotensin-converting enzyme inhibitors on plasma myeloperoxidase in patients with coronary artery disease. Clin Res Cardiol 2010, 100:327-333.

44. ACCORD Study Group, Ginsberg HN, Elam MB, Lovato LC, Crouse JR III, Leiter LA, Linz P: Effects of combination lipid therapy in type 2 diabetes mellitus. N Engl J Med 2010, 362:1563-1574

45. Davis TM, Ting R, Best JD, Donoghoe MW, Drury PL, Sullivan DR, Jenkins AJ, O'Connell RL, Whiting MJ, Glasziou PP, Simes RJ, Kesäniemi YA, Gebski VJ, Scott RS, Keech AC, Fenofibrate Intervention and Event Lowering in Diabetes Study investigators: Effects of fenofibrate on renal function in patients with type 2 diabetes mellitus: the Fenofibrate Intervention and Event Lowering in Diabetes (FIELD) Study. Diabetologia 2011, 54:280-290.

46. Jun M, Zhu B, Tonelli M, Jardine MJ, Patel A, Neal B, Liyanage T, Keech A, Cass A, Perkovic V: Effects of fibrates in kidney disease: a systematic review and meta-analysis. J Am Coll Cardiol 2012, 60:2061-2071.

doi:10.1186/1758-5996-6-30

Cite this article as: Nita et al:: Fenofibrate improves endothelial function and plasma myeloperoxidase in patients with type 2 diabetes mellitus: an open-label interventional study. Diabetology \& Metabolic Syndrome 2014 6:30. 